\title{
Philosophy and Medicine
}

\author{
Volume 119
}

\section{Founding Co-Editor}

Stuart F. Spicker

\section{Senior Editor}

H. Tristram Engelhardt, Jr., Department of Philosophy, Rice University, and Baylor College of Medicine, Houston, TX, USA

\section{Series editors}

Lisa M. Rasmussen, Department of Philosophy, University of North Carolina at Charlotte, Charlotte, NC, USA

\author{
Assistant Editor \\ Jeffrey P. Bishop, Gnaegi Center for Health Care Ethics, Saint Louis University, \\ St. Louis, MO, USA
}

\section{Editorial Board}

George J. Agich, Department of Philosophy, Bowling Green State University, Bowling Green, OH, USA

Nicholas Capaldi, College of Business Administration, Loyola University, New Orleans, LA, USA

Edmund Erde, University of Medicine and Dentistry of New Jersey (Retired), Stratford, NJ, USA

Christopher Tollefsen, Department of Philosophy, University of South Carolina, Columbia, SC, USA

Kevin Wm. Wildes, S.J., President, Loyola University, New Orleans, LA, USA 
The Philosophy and Medicine series is dedicated to publishing monographs and collections of essays that contribute importantly to scholarship in bioethics and the philosophy of medicine. The series addresses the full scope of issues in bioethics, from euthanasia to justice and solidarity in health care. The Philosophy and Medicine series places the scholarship of bioethics within studies of basic problems in the epistemology and metaphysics of medicine. The latter publications explore such issues as models of explanation in medicine, concepts of health and disease, clinical judgment, the meaning of human dignity, the definition of death, and the significance of beneficence, virtue, and consensus in health care. The series seeks to publish the best of philosophical work directed to health care and the biomedical sciences.

More information about this series at http://www.springer.com/series/6414 
Kazem Sadegh-Zadeh

\section{Handbook of Analytic Philosophy of Medicine}

Second Edition

Revised and Enlarged

(120 Figures, 63 Tables)

第 Springer 
Kazem Sadegh-Zadeh

Theory of Medicine

University of Münster

Münster

Germany

ISSN 0376-7418

Philosophy and Medicine

ISBN 978-94-017-9578-4

DOI 10.1007/978-94-017-9579-1
ISSN 2215-0080 (electronic)

ISBN 978-94-017-9579-1 (eBook)

Library of Congress Control Number: 2014958581

Springer Dordrecht Heidelberg New York London

(C) Springer Science+Business Media Dordrecht 2012, 2015

This work is subject to copyright. All rights are reserved by the Publisher, whether the whole or part of the material is concerned, specifically the rights of translation, reprinting, reuse of illustrations, recitation, broadcasting, reproduction on microfilms or in any other physical way, and transmission or information storage and retrieval, electronic adaptation, computer software, or by similar or dissimilar methodology now known or hereafter developed.

The use of general descriptive names, registered names, trademarks, service marks, etc. in this publication does not imply, even in the absence of a specific statement, that such names are exempt from the relevant protective laws and regulations and therefore free for general use.

The publisher, the authors and the editors are safe to assume that the advice and information in this book are believed to be true and accurate at the date of publication. Neither the publisher nor the authors or the editors give a warranty, express or implied, with respect to the material contained herein or for any errors or omissions that may have been made.

Printed on acid-free paper

Springer Science+Business Media B.V. Dordrecht is part of Springer Science+Business Media (www.springer.com) 
Maria, David, and Manuel 


\section{Foreword}

This work will shape the philosophy of medicine for years to come. There are very few scholarly endeavors that truly encompass a field while also having the promise of shaping and changing that field. Kazem Sadegh-Zadeh has produced such a volume for the philosophy of medicine. This is a foundational work of amazing depth and scope, which is also user-friendly. No one engaged in the philosophy of medicine will in the future be able to proceed, save in the light of and in response to the analyses, arguments, and reflections Sadegh-Zadeh has compassed in this extraordinarily rich and important study. He has succeeded in bringing together in an integrated vision an exploration of the epistemological, practical, and logical frameworks that sustain the engagement of physicians, as well as define the place of patients in medicine. This opus magnum provides remarkably careful explorations of the concept of disease, as well as of the diagnosis and treatment of patients in the acts of medical knowing and treatment. It situates the intertwining of diagnoses, the appreciation of therapy warrants, and the engagement of physicians in treating patients within the complex phenomenon of medicine. This work even has what is tantamount to an appendix that shows the bearing of logic on medicine.

The work begins with a careful exploration of the language of medicine, attending to its epistemic impact, its syntax, semantics, and pragmatics, including the various ways in which medical concepts are framed and engaged. Sadegh-Zadeh then examines medicine's encounter with the patient as a biopsycho-social reality caught up in the drama of health, illness, and disease. In this study of medical practice, Sadegh-Zadeh creatively attends to the interaction of patient and physician in clinical practice. His analysis of the interconnection of anamnesis, diagnosis, prognosis, therapy, and prevention in the clinical context is innovative, displaying a remarkable depth of understanding that constitutes not just a foundational contribution to the literature, but a reframing of the field. It offers a comprehensive perspective, which is a tour de force. Drawing on a nuanced and subtle appreciation of epistemology in general and an account of the character of medicine in particular, Sadegh- 
Zadeh explores the semantics and pragmatics of medical knowledge. He then relates these reflections to the intertwining of moral concerns, the character of logic in medicine, and a consideration of medical ontology within which, among other things, he provides a careful analysis of medical reality and the character of medical truth. He ties all of this to what can only be described as a powerful vision of the conceptual fundamentals that constitute the scope of the philosophy of medicine. Sadegh-Zadeh offers what will without doubt for the foreseeable future be the most widely influential and comprehensive account of the philosophy of medicine.

This impressively nuanced work bears the mark of a lifetime of research, reflections, and publications on the philosophy of medicine. While others were engaged in the birth of bioethics, Sadegh-Zadeh was focusing with critical energy on the philosophy of medicine. As a result, he became one of the central figures driving the re-emergence of the philosophy of medicine as a scholarly field. Early on, he helped to establish and then expand the scope and depth of philosophical medicine. One must note in particular that he aided in supporting scholarship in the philosophy of medicine through his pioneering work with his journal Metamed which was established in 1977, and which then later took the name Metamedicine and which finally became Theoretical Medicine. He has also been involved from the early years in The Journal of Medicine and Philosophy. Both through his own scholarly articles, as well as through creating vehicles for the publication of scholarly articles, his work in the philosophy of medicine has helped locate bioethics within the broader geography of the foundational explanation and therapeutic concerns that define medicine. As a physician and philosopher, Sadegh-Zadeh has without flinching addressed the conceptually challenging issues that lie at the basis of a philosophical appreciation of contemporary medicine. The result is that Kazem Sadegh-Zadeh has come to have a command of the philosophy of medicine possessed by no other scholar.

Drawing on a rich lifetime of scholarship, Sadegh-Zadeh has been able to integrate recent work in epistemology, the philosophy of science, and logic in a work in the philosophy of medicine. Because of his disciplined and innovative eye, this volume sheds a bright analytic light on the character of contemporary medicine and charts the future of the philosophy of medicine. It is marked both by creativity and an encyclopedic scope, and will establish itself as the standard for the field. It is likely that no one could have accomplished such a substantial exploration of the nature of medicine, other than Kazem Sadegh-Zadeh. He has produced an indispensable resource for scholars in the philosophy of medicine, including those working in bioethics. This work surely secures Kazem Sadegh-Zadeh's place as a cardinal founder of the contemporary field of the philosophy of medicine.

Houston, TX

April 23, 2011
H. Tristram Engelhardt, Jr. Professor, Rice University Professor Emeritus, Baylor College of Medicine 


\section{Preface to the Second Edition}

This is a book for those who understand it, or are willing to do so, but not for those who don't understand it. So, reviews and public comments by members of the latter category are unwelcome. The volume is a carefully revised and considerably enlarged new edition. Type-setting errors and other bugs have been corrected and three flawed figures have been redrawn. Other changes made include some stylistic amendments and the following additions and expansions, which have increased the number of parts from VIII to IX, of chapters from 30 to 34 , of figures by 4 , of tables by 2 , and of pages by 91 :

Added:

- Sections 3.4-3.5: The Pragmatic Impact of Medical Language + The Communal Origin of Medical Language (pp. 58-59),

- Chapter 4: Medical Linguistics (pp. 61-68),

- Subsection 17.4.4: Probabilistic Networks (pp. 626-627),

- Part VI: Medical Artificial Intelligence (pp. 709-743).

Expanded:

- Subsubsection "Free will": (pp. 156-162),

- Section 9.1.2: Dynamic, Branching Clinical Questionnaires (301-312),

- Section 17.3: Modal Logics in Medicine (pp. 614-619),

- Subsection 17.5.4: Fuzzy Logic in Biomedicine (pp. 665-683),

- Section 24.3: Misdiagnoses (pp. 821-826),

- Chapter 27: Perspectivism (pp. 875-884),

- Subsection 33.1.5: Bayes's Theorem (pp. 1050-1053),

- Subsection 34.2.4: Degrees of Fuzziness and Clarity (pp. 1079-1083),

- Section 34.5: Summary (pp. 1114-1120).

Tecklenburg,

Germany 49545

October 15, 2013
Kazem Sadegh-Zadeh

Emeritus Professor of Philosophy of Medicine University of Münster, Germany 


\section{Preface to the First Edition}

Medicine is a science and practice of intervention, manipulation, and control concerned with curing sick people, caring for sick people, preventing maladies, and promoting health. What necessitates this task, is the human suffering that results from maladies, and the desire for remedy and relief. Medicine serves this human need by attempting to lessen suffering that human beings evaluate as bad, and to restore and augment well-being that human beings evaluate as good. On this account, medicine as health care is practiced morality insofar as it acts against what is bad, and promotes what is good, for human beings. And insofar as it seeks rules of action toward achieving those goals and strives continually to improve the quality and efficacy of these rules, i.e., as clinical research, it belongs to normative ethics. Medicine is not human biology, biophysics, biochemistry, or biopathology. Nor is it any sum of these and similar biomedical and natural sciences. To view it as such, would shift medicine toward bio- and anthropotechnology where morality and ethics would lose their meaning and significance. As an aid in preventing such an autolysis of medicine, the present book elucidates and advances the view sketched above by:

- analyzing the structure of medical language, knowledge, and theories,

- inquiring into the foundations of the clinical encounter,

- introducing the logic and methodology of clinical decision-making,

- suggesting comprehensive theories of organism, life, and psyche; of health, illness, and disease; and of etiology, diagnosis, prognosis, prevention, and therapy,

- investigating the moral and metaphysical issues central to medical practice and research.

To this end, the book offers in its final Part IX, as an appendix so to speak, a concise introduction to some focal systems and methods of logic that are needed and used throughout. Each line, paragraph, and page of its remaining eight parts relies upon what precedes it and what has been said in Part IX. 
The readers, therefore, should study the book systematically following the instructions given in Figure 1 on page 9. In that case, it will prove absolutely self-contained. It does not require any special knowledge and is easily accessible to all interested students. By virtue of its didactic style, the book is also usable in graduate courses in the philosophy of medicine, bioethics, medical ethics, philosophy, medical artificial intelligence, and clinical decision-making.

My thanks are due to H. Tristram Engelhardt, Jr., from whom I have learned, among many other things, that the concept of disease says what ought not to be (Engelhardt, 1975, 127). It is thus a deontic concept (from

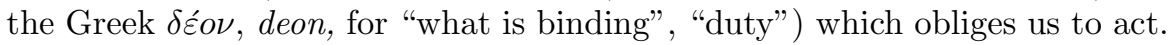
Since this normative aspect is dismissed by most physicians and philosophers of medicine alike, initially I wanted to analyze and demonstrate it in what eventually became the present handbook, HAPM, by means of deontic logic. In the process of writing, however, my thoughts extended beyond the concept of disease to the entire field of medicine when I fully recognized the deonticity of the field as a whole in the early 1980s (Sadegh-Zadeh, 1983). Although it is a fascinating feature of medicine that places the institution of health care in the same category as charity, it seems to have been overlooked by philosophers of medicine and medical ethicists until now. I hope they will concern themselves with this issue and discover additional facts about it when they read HAPM.

Also, my intellectual debt is to four scholars whose works greatly impacted my way of thinking and my life: Karl Eduard Rothschuh (1908-1984), one of my teachers at the University of Münster in Germany, ignited my love for the philosophy of medicine in 1964 when I was a graduate student of medicine and philosophy; Patrick Suppes's precision in philosophizing taught me analytic philosophy in the late 1960s; Newton C.A. da Costa's paraconsistent logic changed my view of logic and my Weltanschauung in the late 1970s; and Lotfi A. Zadeh's fuzzy logic changed everything anew and inspired me to initiate fuzzy analytic philosophy and methodology of medicine in the early 1980s.

I am particularly grateful to my wife, Maria, for surrounding me with so much love and support over the long period of creating HAPM; and to my sons, David and Manuel, for their assistance. Manuel drew the figures. David did extensive LATEX work (references, indexes) and produced, with the aid of Matlab ${ }^{\circledR}$, the 3D representation of high blood pressure on page 696 .

I would also like to extend special thanks to the editors of the Philosophy and Medicine for including HAPM in their highly respectable book series, and for excellent supervision, advice, and support; to Mr. Richard Preville in Charlotte, North Carolina, for carefully transforming my imperfect 'German English' into well-readable English; and to Springer for their outstanding production process management. But without the patient and competent work of three anonymous reviewers, none of us would be reading this line right now. I wholeheartedly thank all of them for their thoughtful comments and valuable suggestions.

Some of the ideas in this handbook present a further development of their seeds and preliminary forms that have appeared in my previous publications. 
Specifically, my theories of health and disease in Section 7.3, of etiology in Section 7.5, and of diagnosis in Section 9.2 are based on my "Fundamentals of clinical methodology", 1-4, in Artificial Intelligence in Medicine (19942000); on my theory of fuzzy health, illness, and disease in The Journal of Medicine and Philosophy (2000, 2008); and on my "The logic of diagnosis" in Handbook of the Philosophy of Science, Vol. 16 (2011). Section 17.5.4 relies on my previous articles "Fuzzy genomes" and "The fuzzy polynucleotide space revisited" in Artificial Intelligence in Medicine (2000, 2007). Although during the process of writing the handbook, I have drawn on this previously published work, most of this material has been substantially revised, rewritten, and supplemented.

One of the reviewers proposed that I create a companion website for HAPM, which could provide a glossary and additional resources online. I welcomed the proposal, as I have already been offering a website on philosophy of medicine in German for many years. This website has now been internationalized to facilitate studies in the analytic philosophy of medicine, including HAPM. You may take a look at it here $\Rightarrow$ http://www.philmed-online.net

Tecklenburg,

Germany 49545

Summer 2010
Kazem Sadegh-Zadeh

Emeritus Professor of Philosophy of Medicine University of Münster, Germany 


\section{Contents}

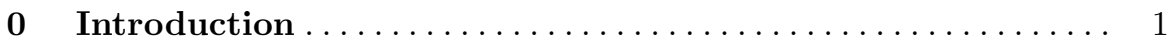

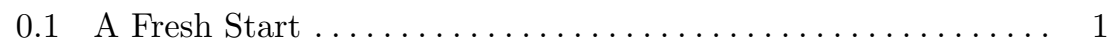

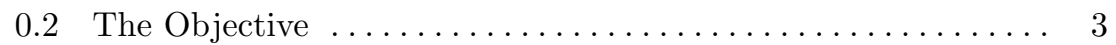

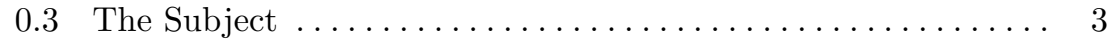

0.4 Methods of Inquiry $\ldots \ldots \ldots \ldots \ldots \ldots \ldots \ldots \ldots \ldots \ldots \ldots \ldots$

0.5 How to Read this Book $\ldots \ldots \ldots \ldots \ldots \ldots \ldots \ldots \ldots$

\section{Part I The Language of Medicine}

1 The Epistemic Impact of Medical Language $\ldots \ldots \ldots \ldots \ldots$

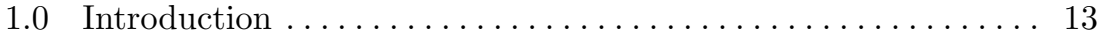

1.1 Types of Knowledge .......................... 15

1.2 Propositional Knowledge . ....................... 17

1.3 Propositions and Facts .......................... 19

1.4 Medical Sentences and Statements .................. 21

1.5 Medical Concepts .......................... 25

1.6 How to Care About our Medical Concepts? .............. 28

1.7 Summary .............................. 30

2 The Syntax and Semantics of Medical Language .......... 31

2.0 Introduction ............................. 31

2.1 Medical Language is an Extended Natural Language ........ 32

2.2 What a Medical Term Means ....................... 33

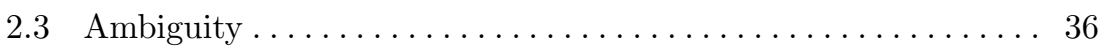

2.4 Vagueness .................................. 37

2.4.1 The Nature of Vagueness . . . . . . . . . . . . . . 37

2.4.2 The Sorites Paradox ....................... 42

2.4 .3 Varieties of Vagueness $\ldots \ldots \ldots \ldots \ldots \ldots \ldots \ldots \ldots . \ldots \ldots$

2.5 Clarity and Precision $\ldots \ldots \ldots \ldots \ldots \ldots \ldots \ldots \ldots \ldots \ldots \ldots \ldots$

2.6 Semantic Nihilism ............................. 48 


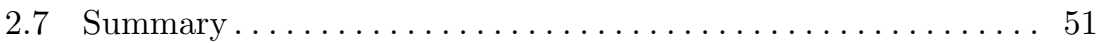

3 The Pragmatics of Medical Language ................ 53

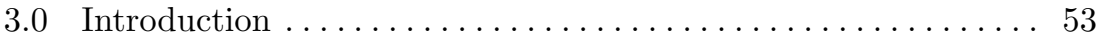

3.1 The So-Called Language Games . . . . . . . . . . . . . . . 53

3.2 Assertion, Acceptance, and Rejection .............. 54

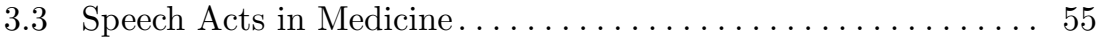

3.3 .1 Constatives . . . . . . . . . . . . . . . . . . . . 56

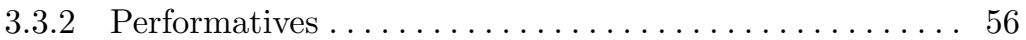

3.4 The Pragmatic Impact of Medical Language . . . . . . . . . . . . . . 58

3.5 The Communal Origin of Medical Language . . . . . . . . . . . . . 59

3.6 Summary ............................ 60

4 Medical Linguistics $\ldots \ldots \ldots \ldots \ldots \ldots \ldots \ldots \ldots \ldots \ldots \ldots \ldots \ldots$

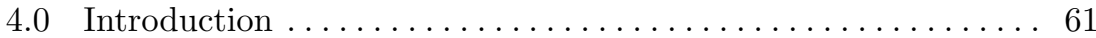

4.1 What Medical Linguistics is $\ldots \ldots \ldots \ldots \ldots \ldots \ldots \ldots \ldots \ldots \ldots$

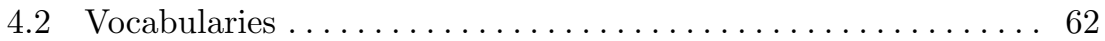

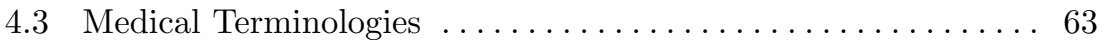

4.3.1 Medical Nomenclatures ... . . . . . . . . . . . . . 64

4.3.2 International Classification of Diseases (ICD) . . . . . 65

4.3.3 Systematized Nomenclature of Medicine (SNOMED) . . 67

4.3.4 GALEN, GRAIL, UMLS, MeSH, et al. . . . . . . . . 67

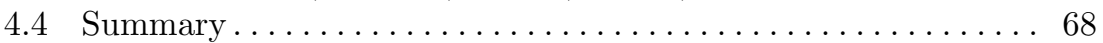

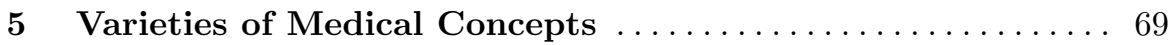

5.0 Introduction . . . . . . . . . . . . . . . . . . . . . . . 69

5.1 Qualitative, Comparative, and Quantitative Concepts . . . . . . 70

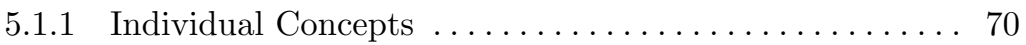

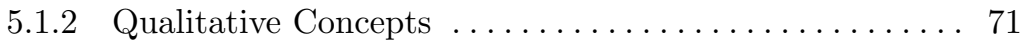

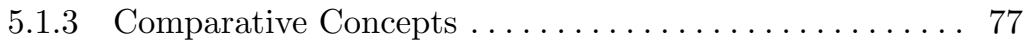

5.1 .4 Quantitative Concepts .................. 82

5.2 Dispositional Terms in Medicine ................. 88

5.3 Linguistic and Numerical Variables in Medicine . . . . . . . . . 90

5.4 Non-Classical vs. Classical Concepts . . . . . . . . . . . . . . 91

5.5 Summary ......................... 92

6 Fundamentals of Medical Concept Formation ............ 93

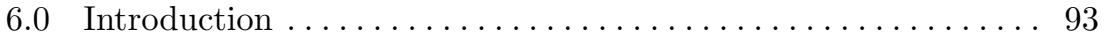

6.1 What a Definition is . . . . . . . . . . . . . . . . . . 94

6.2 What Role a Definition Plays ... . . . . . . . . . . . . 96

6.3 Methods of Definition . . . . . . . . . . . . . . . . . . . . . . . . 97

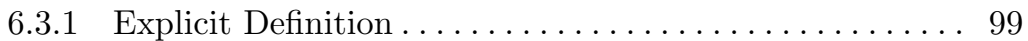

6.3 .2 Conditional Definition ....................... 101

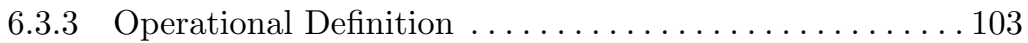

6.3.4 Definition by Cases ................... 106 


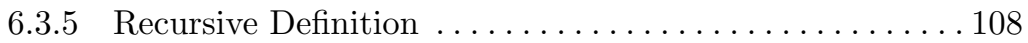

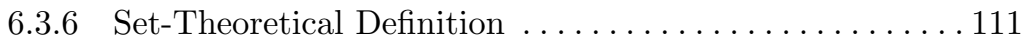

6.3 .7 Ostensive Definition . . . . . . . . . . . . . . . . . . 113

6.4 What an Explication is . . . . . . . . . . . . . . 115

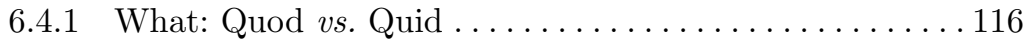

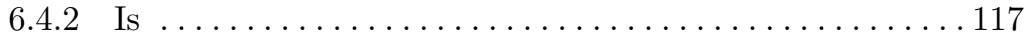

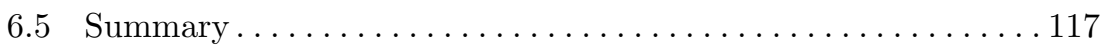

\section{Part II Medical Praxiology}

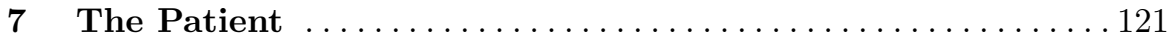

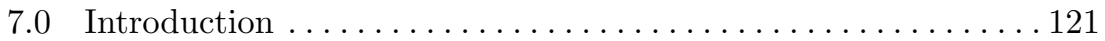

7.1 The Suffering Individual . . . . . . . . . . . . . . . . . . . 123

7.2 The Bio-Psycho-Social Agent . . . . . . . . . . . . . . . 124

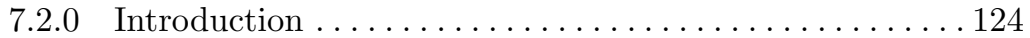

7.2 .1 The Living Body . . . . . . . . . . . . . . . . . 124

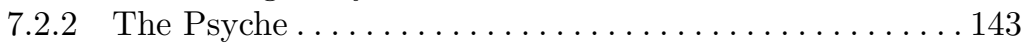

7.2 .3 The Social Agent . . . . . . . . . . . . . . . . . . . 154

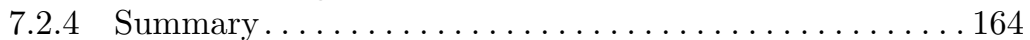

7.3 Health, Illness, and Disease . . . . . . . . . . . . . . 165

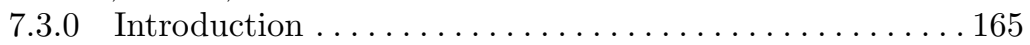

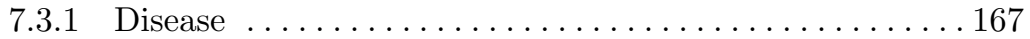

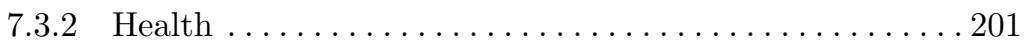

7.3 .3 Illness . . . . . . . . . . . . . . . . . . . 204

7.3.4 Disease, Health, and Illness Violate Classical Logic . . . 210

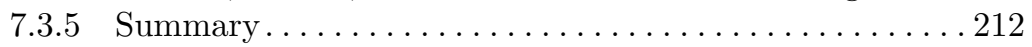

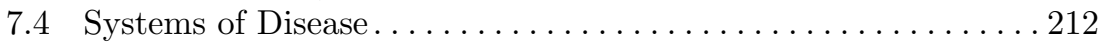

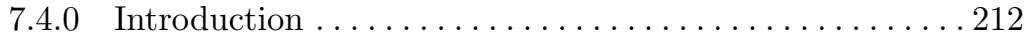

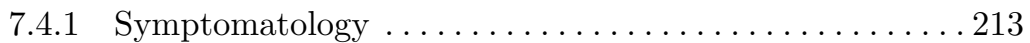

7.4 .2 Nosological Systems . . . . . . . . . . . . . . . . . . . 219

7.4 .3 Pathology vs. Nosology . . . . . . . . . . . . . . . . . 220

7.4 .4 Nosological Spaces . . . . . . . . . . . . . . . . . . . 225

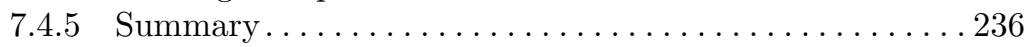

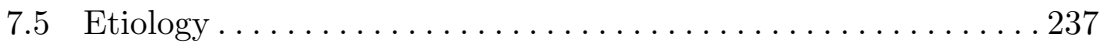

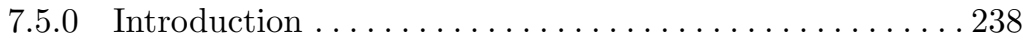

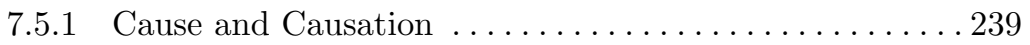

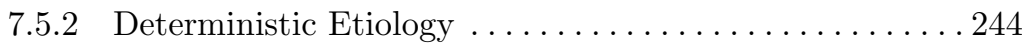

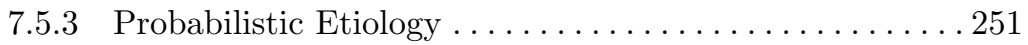

7.5 .4 Fuzzy Etiology . . . . . . . . . . . . . . . . . . . . . 282

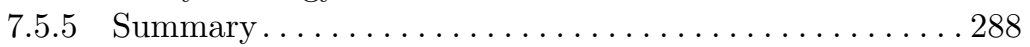

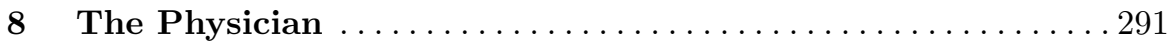




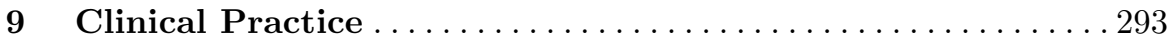

9.0 Introduction . . . . . . . . . . . . . . . . . . . . . . . . . . . . 293

9.1 The Clinical Encounter. . . . . . . . . . . . . . . . . . . . . . . 293

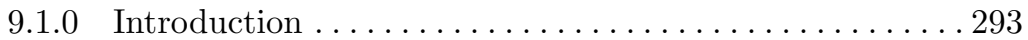

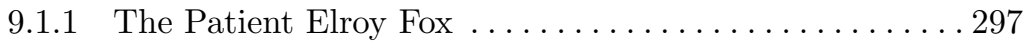

9.1.2 Dynamic, Branching Clinical Questionnaires ......... 301

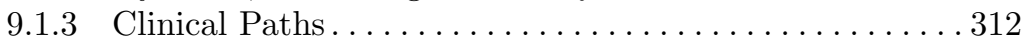

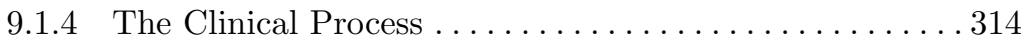

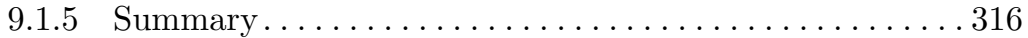

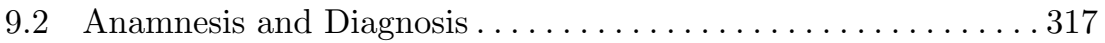

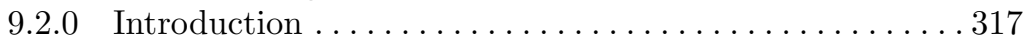

9.2.1 The Clinical Goal . . . . . . . . . . . . . . . . . . . 317

9.2.2 The Logical Structure of Medical Statements . . . . . . . . 324

9.2.3 Action Indication and Contra-Indication . . . . . . . . . . 326

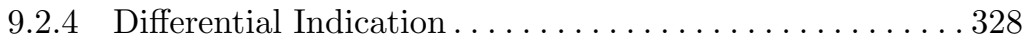

9.2.5 The Computability of Differential Indication . . . . . . . . 334

9.2.6 The Logical Structure of Diagnosis . . . . . . . . . . . . 337

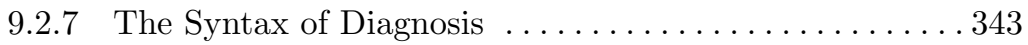

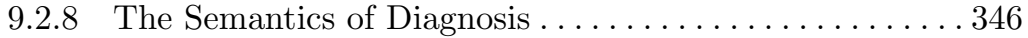

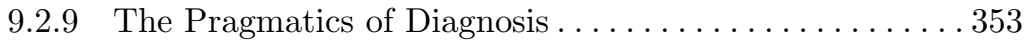

9.2.10 The Methodology of Diagnostics . . . . . . . . . . . 357

9.2.11 The Logic of Diagnostics . . . . . . . . . . . . . . . 359

9.2.12 The Epistemology of Diagnostics . . . . . . . . . . . . . . 359

9.2.13 The Relativity of Diagnosis . . . . . . . . . . . . . . . 364

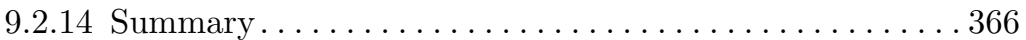

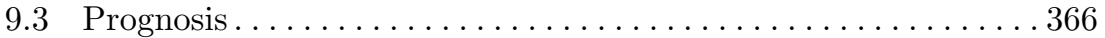

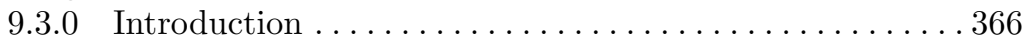

9.3.1 The Clinical Role of Prognosis . . . . . . . . . . . . . 367

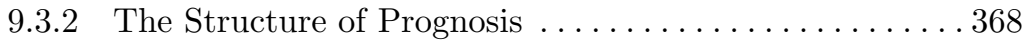

9.3.3 The Uncertainty of Prognosis . . . . . . . . . . . . . . . . . 369

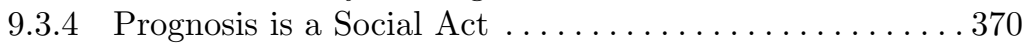

9.3.5 Summary............................ 370

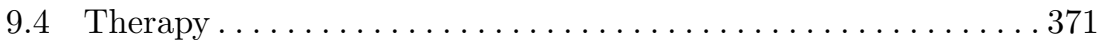

9.4 .0 Introduction $\ldots \ldots \ldots \ldots \ldots \ldots \ldots \ldots \ldots \ldots \ldots \ldots \ldots \ldots$

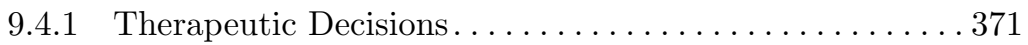

9.4.2 Expected Value Therapeutic Decision-Making . . . . . . . 373

9.4.3 Treatment Threshold Probability ............... 376

9.4.4 Treatments are Social Acts . . . . . . . . . . . . . . . . . . . . . . . . 377

9.4.5 Therapeutic Efficacy . . . . . . . . . . . . . . . . 377

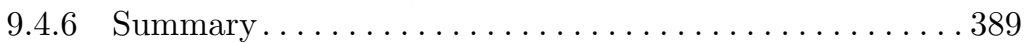

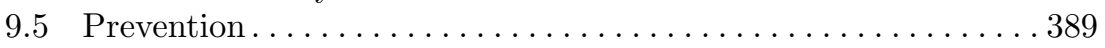

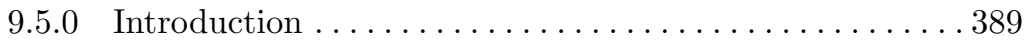

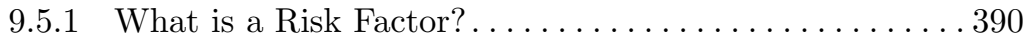

9.5.2 Prevention is Goal-Driven Practice . . . . . . . . . . . 397

9.5 .3 Summary......................... 397 


\section{Part III Medical Epistemology}

10 The Architecture of Medical Knowledge ................. 401

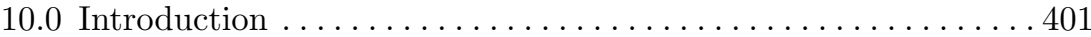

10.1 Detachment of Medical Knowledge from the Knower ......... 402

10.2 The Syntax of Medical Knowledge .................... 405

10.2.1 Problematic Sentences . . . . . . . . . . . . . . . . . . . 406

10.2.2 First-Order Sentences . . . . . . . . . . . . . . . . . . . . . . 407

10.2.3 Modal Sentences . . . . . . . . . . . . . . . . . . . . . 408

10.2.4 Probabilistic Sentences ........................ 408

10.2.5 Fuzzy Sentences .............................. 409

10.3 Medical Hypotheses. . . . . . . . . . . . . . . . . . . . . . . . . . . . 413

10.4 Theories in Medicine............................. 417

10.4.1 The Statement View of Theories ................418

10.4.2 The Non-Statement View of Theories . . . . . . . . . . 421

10.4.3 The Semantic View of Theories ...............447

10.4.4 Theory-Nets and Intertheoretic Relations ............. 447

10.4.5 Untestability of Theories . . . . . . . . . . . . . . . . . . 457

10.4.6 Theories Fuzzified ....................... 458

10.5 Summary . . . . . . . . . . . . . . . . . . . . . . 460

11 Types of Medical Knowledge $\ldots \ldots \ldots \ldots \ldots \ldots \ldots \ldots \ldots \ldots 4$

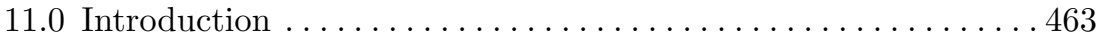

11.1 Shallow and Deep Medical Knowledge................. 464

11.2 Classificatory Knowledge .............................. 464

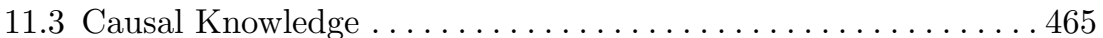

11.4 Experimental Knowledge ......................... 467

11.5 Theoretical Knowledge ........................... 470

11.6 Practical Knowledge . . . . . . . . . . . . . . . . . . . . . . . . 471

11.7 Clinical Knowledge .......................... 476

11.8 Medical Metaknowledge .......................... 478

11.9 Summary .................................. 478

12 The Semantics and Pragmatics of Medical Knowledge .... 479

12.0 Introduction . . . . . . . . . . . . . . . . . . . . . . . 479

12.1 Justified True Belief . .......................... 480

12.1.1 Truth ................................480

12.1.2 Justification ........................487

12.1.3 Are There Justified True Beliefs in Medicine? ......... 506

12.2 Realism ....................................508

12.2.1 Metaphysical Realism ......................508

12.2.2 Semantic Realism .......................... 509

12.2.3 Epistemic Realism ........................... . 509

12.2.4 Medical Realism ......................... 510 
12.3 Anti-Realism. . . . . . . . . . . . . . . . . . . . . . . . 512

12.3.1 Metaphysical Anti-Realism . . . . . . . . . . . . . . . . . . . . . . . . . . 512

12.3.2 Semantic Anti-Realism . . . . . . . . . . . . . . . . . 513

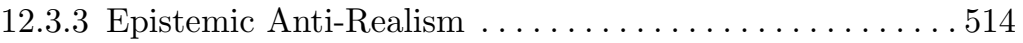

12.3.4 Medical Anti-Realism . . . . . . . . . . . . . . . . . 514

12.4 Beyond Realism and Anti-Realism in Medicine . . . . . . . . . . 516

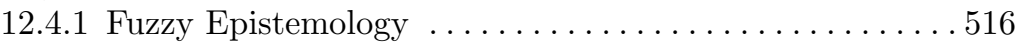

12.4.2 Constructivism . . . . . . . . . . . . . . . . . . 517

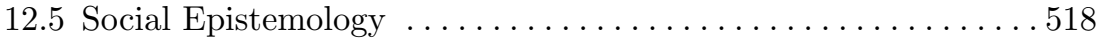

12.5.1 Logical Empiricism and Critical Rationalism . . . . . . . 519

12.5.2 The Rise of Social Epistemology . . . . . . . . . . . . 522

12.5.3 Medical Knowledge is a Social Status . . . . . . . . . . . 532

12.5.4 Social Constructivism . . . . . . . . . . . . . . . . . 543

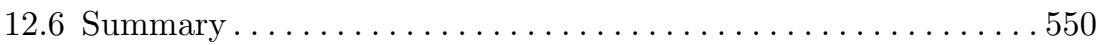

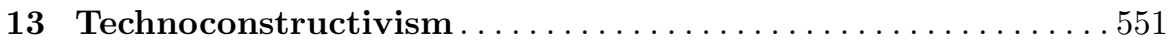

13.0 Introduction . . . . . . . . . . . . . . . . . . . . . . . . . . 551

13.1 Experiments as Epistemic Assembly Lines . . . . . . . . . . . . . . 552

13.2 Epistemic Machines. . . . . . . . . . . . . . . . . . . . 555

13.2.1 An Experiment is a Production System . . . . . . . . . . 557

13.2.2 An Experiment is an Epistemic Machine . . . . . . . . . 559

13.3 Epistemic Factories . . . . . . . . . . . . . . . . . . . . 562

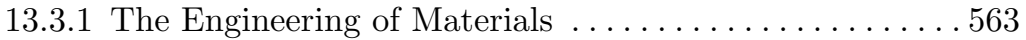

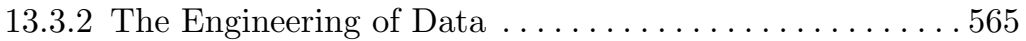

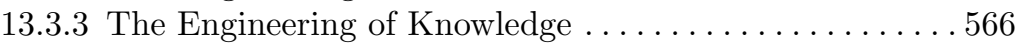

13.4 The Global Knowledge-Making Engine . . . . . . . . . . . . . . . 568

13.5 The Industrialization of Knowledge . . . . . . . . . . . . . . 568

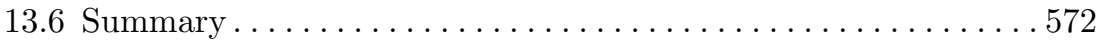

\section{Part IV Medical Deontics}

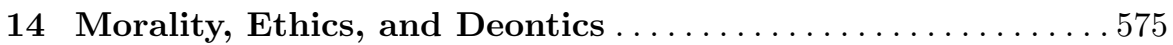

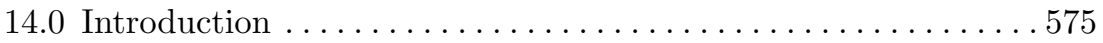

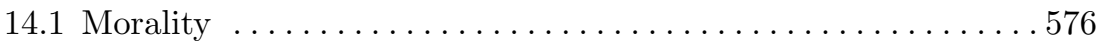

14.2 Ethics and Metaethics ..................... 578

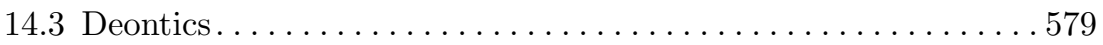

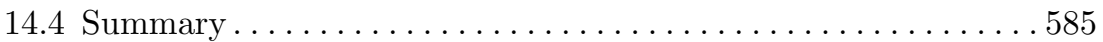

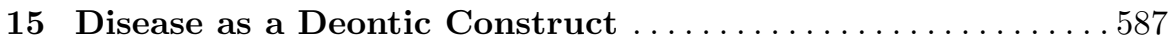

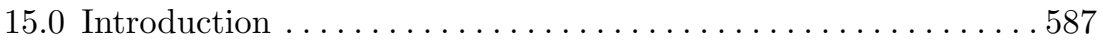

15.1 Common Morality . . . . . . . . . . . . . . . . . 587

15.2 Common Morality as a Deontic-Social Institution . . . . . . . . 588

15.3 Deontic Sets . . . . . . . . . . . . . . . . . . . . . . . . . . 589

15.4 The Deontic Construction of Prototype Diseases. . . . . . . . . . . 592 
15.5 Summary ........................... 595

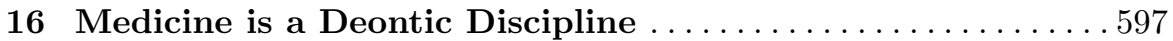

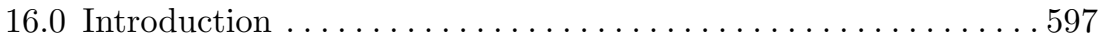

16.1 Deonticity in Clinical Practice . . . . . . . . . . . . . . . . . . 598

16.2 Deonticity in Medical Research . . . . . . . . . . . . . . . . 601

16.3 Deontic Things in Medicine . . . . . . . . . . . . . . . . . . 602

16.4 Summary . . . . . . . . . . . . . . . . . . . . 602

\section{Part V Medical Logic}

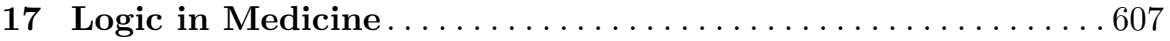

17.0 Introduction . . . . . . . . . . . . . . . . . . . . . . . 607

17.1 Classical Logic in Medicine . . . . . . . . . . . . . . . . . . 609

17.2 Paraconsistent Logic in Medicine . . . . . . . . . . . . . . 613

17.3 Modal Logics in Medicine . . . . . . . . . . . . . . . . . . . . 614

17.3.1 Alethic Modal Logic in Medicine . . . . . . . . . . . 615

17.3.2 Deontic Logic in Medicine . . . . . . . . . . . . . 615

17.3.3 Epistemic Logic in Medicine . . . . . . . . . . . . 616

17.3.4 Temporal Logic in Medicine . . . . . . . . . . . . . 618

17.4 Probability Logic in Medicine . . . . . . . . . . . . . . . . . . . 619

17.4.1 Uncertainty and Randomness . . . . . . . . . . . 619

17.4.2 Probabilistic-Causal Analysis . . . . . . . . . . . . . 6621

17.4.3 Probabilistic-Causal Factors . . . . . . . . . . . . 625

17.4.4 Probabilistic Networks . . . . . . . . . . . . . . 626

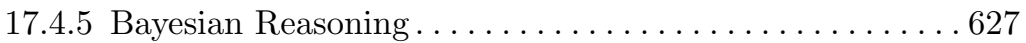

17.5 Fuzzy Logic in Medicine . . . . . . . . . . . . . . . . . . . . . . 628

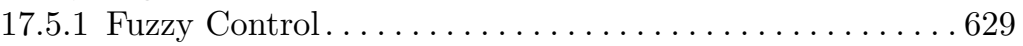

17.5.2 Fuzzy Clinical Decision-Making . . . . . . . . . . . . . 640

17.5.3 Similaristic Reasoning in Medicine ..............663

17.5.4 Fuzzy Logic in Biomedicine . . . . . . . . . . . . . 665

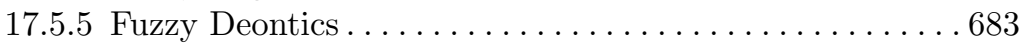

17.5.6 Fuzzy Concept Formation in Medicine . . . . . . . . . . 689

17.6 Summary ........................... 698

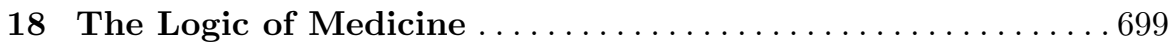

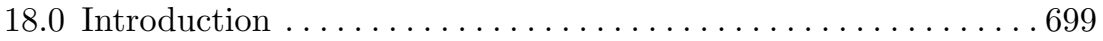

18.1 What is Logic? . . . . . . . . . . . . . . . . . . . . . 699

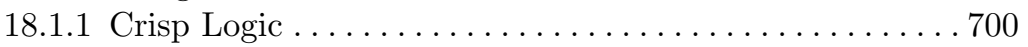

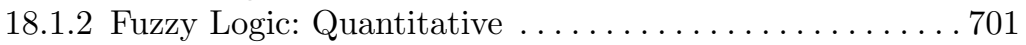

18.1.3 Fuzzy Logic: Qualitative . . . . . . . . . . . . . . . 701

18.2 Implication Structures . . . . . . . . . . . . . . . . . . 701

18.3 On the Logic of Medicine . . . . . . . . . . . . . . . . . 702

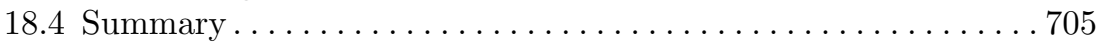




\section{Part VI Medical Artificial Intelligence}

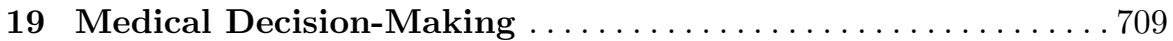

19.0 Introduction .................................. 709

19.1 In the Beginning was the Punched Card ............... 710

19.2 Then Came Probability Theory and Decision Theory........ 711

19.3 And a New Field of Research and Practice Emerged ......... 712

19.4 But Probabilities Soon Became Intractable .............. 713

19.5 Summary . . . . . . . . . . . . . . . . . . . . . . 713

20 Clinical Decision Support Systems ................... 715

20.0 Introduction .................................... 715

20.1 Medical Expert Systems ........................ 716

20.1.1 MYCIN, CASNET, INTERNIST-1, QMR, DXplain . . . 717

20.1.2 The Architecture of an Expert System .............. 719

20.1.3 Types of Medical Expert Systems . . . . . . . . . . . . 722

20.2 Neural Computation ............................ 724

20.2.1 Natural Neural Networks ..................... 724

20.2.2 Artificial Neural Networks (ANNs) . . . . . . . . . . 726

20.3 Evolutionary Computation ........................ 728

20.4 Hybrid Decision Support Systems . . . . . . . . . . . . . . 729

20.5 Hospital Information Systems . . . . . . . . . . . . . . . . . . 731

20.6 Summary . . . . . . . . . . . . . . . . . . . . . . . 732

21 Artificial Intelligence in Medicine? .................. 733

21.0 Introduction . . . . . . . . . . . . . . . . . . . . . . . . . 733

21.1 Natural Intelligence ............................. 733

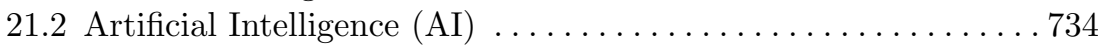

21.2.1 Aims and Scope of AI ...................... 735

21.2 .2 Limitations of AI .......................... 739

21.2.3 Is 'AI in Medicine' possible? .................... 742

21.3 Summary .................................. 743

\section{Part VII Medical Metaphysics}

22 On What There Are $\ldots \ldots \ldots \ldots \ldots \ldots \ldots \ldots \ldots \ldots \ldots \ldots 74$

22.0 Introduction ................................ 747

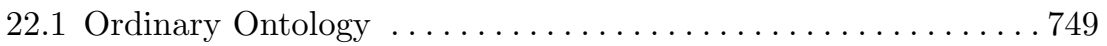

22.1.1 Pure Ontology ............................. 750

22.1.2 Applied Ontology . ...................... 760

22.1.3 Formal Ontology ........................ 760

22.2 Fuzzy Ontology . . . . . . . . . . . . . . . . . . . 761

22.3 Vague, Fictional, and Non-Existent Entities............. 767 


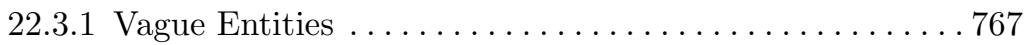

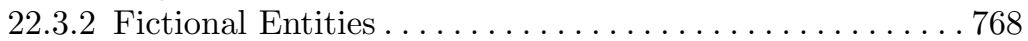

22.3.3 Non-Existent Entities . . . . . . . . . . . . . . . . . . . . 771

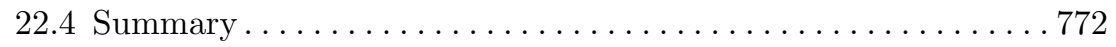

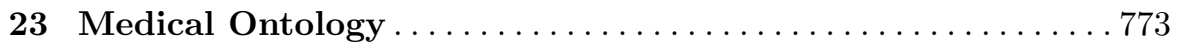

23.0 Introduction . . . . . . . . . . . . . . . . . . . . . . . . . . . 773

23.1 The Ontology of Medical Knowledge . . . . . . . . . . . . . 774

23.1.1 Ontological Commitments of Medical Knowledge . . . . . 774

23.1.2 Medically Relevant Ontological Categories . . . . . . . . . 776

23.1.3 Models for Medical Knowledge . . . . . . . . . . . . 777

23.2 Clinical Ontology . . . . . . . . . . . . . . . . . . . . . 778

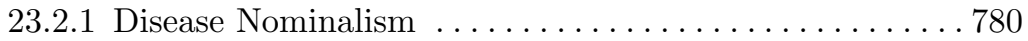

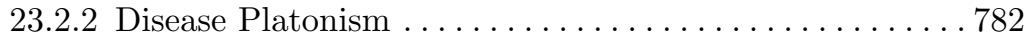

23.2.3 Disease Tropism . . . . . . . . . . . . . . . . . . . . . . . . . . . . . . . 784

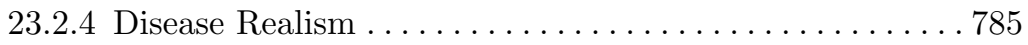

23.3 The Ontology of Psychiatry and Psychosomatics . . . . . . . . 786

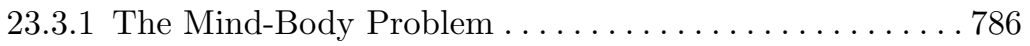

23.3.2 Mental States . . . . . . . . . . . . . . . . . . 791

23.3.3 The Ontology of Mental Diseases . . . . . . . . . . . . 792

23.3.4 The Ontology of Psychosomatic Diseases . . . . . . . . 795

23.4 Biomedical Ontology Engineering . . . . . . . . . . . . . . 797

23.5 Formal Medical Ontology . . . . . . . . . . . . . . . . . 800

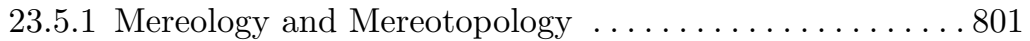

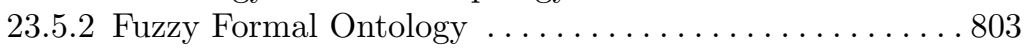

23.6 Medical Ontology de re and de dicto ............... 815

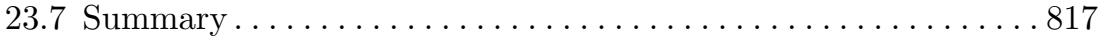

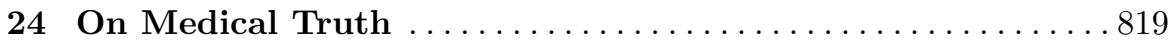

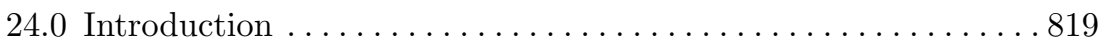

24.1 Truth in Medical Sciences . . . . . . . . . . . . . . . . . 819

24.2 Truth in Clinical Practice . . . . . . . . . . . . . . . . . 820

24.3 Misdiagnoses. . . . . . . . . . . . . . . . . . . . . . 821

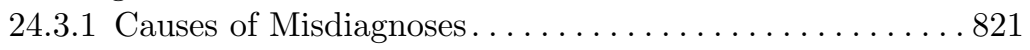

24.3.2 The Concept of Misdiagnosis . . . . . . . . . . . . . 823

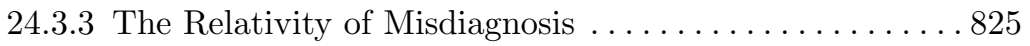

24.3.4 Diagnoses are Hypotheses . . . . . . . . . . . . . . 8 825

24.3.5 Performative Diagnostic Truth . . . . . . . . . . . . . 826

24.4 Truth Made in Medicine . . . . . . . . . . . . . . . . . . . . 826

24.5 Summary . . . . . . . . . . . . . . . . . . . . . . . 827 


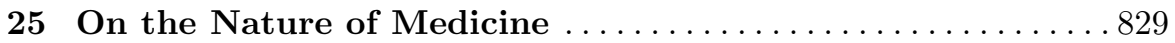

25.0 Introduction . . . . . . . . . . . . . . . . . . . . . . . . . . . 829

25.1 The Subject and Goal of Medicine . . . . . . . . . . . . . . 830

25.2 Is Medicine a Natural Science? . . . . . . . . . . . . . . . . . . . . 832

25.3 Is Medicine an Applied Science? . . . . . . . . . . . . . . . . 833

25.4 Does Medicine Belong to the Humanities? . . . . . . . . . . . . . . . . 834

25.5 Is Medicine a Practical Science? . . . . . . . . . . . . . . . . . . . 834

25.5.1 Practical vs. Theoretical Sciences ............... 834

25.5.2 Means-End Research . . . . . . . . . . . . . . . . . . 835

25.5.3 Clinical Research is a Practical Science........... . 836

25.5.4 Relationships Between Biomedicine and Clinical Medicine 842

25.6 Medicine is Practiced Morality as well as Ethics . . . . . . . . . . 843

25.6.1 Clinical Practice is Practiced Morality . . . . . . . . . . . 843

25.6.2 Clinical Research is Normative Ethics . . . . . . . . . . . . . 844

25.7 Quo Vadis Medicina? . . . . . . . . . . . . . . . . . . . 845

25.7.1 Medicine is in Transition to an Engineering Science . . . 846

25.7.2 Medicine Toward Anthropotechnology and Posthumanity 851

25.8 Summary . . . . . . . . . . . . . . . . . . . . . 852

\section{Part VIII Epilog}

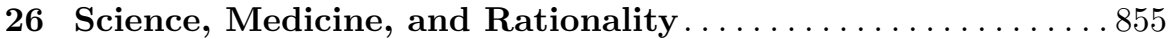

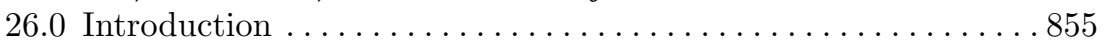

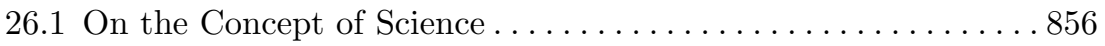

26.1.1 Research Institutions $\ldots \ldots \ldots \ldots \ldots \ldots \ldots \ldots \ldots \ldots$

26.1.2 Scientific Research Fields . . . . . . . . . . . . . . . 860

26.1.3 Science in General . . . . . . . . . . . . . . . . . . . . . . . . . . . . . . . 862

26.1.4 Types of Science . . . . . . . . . . . . . . . . . . 864

26.2 On the Scientific Status of Medicine . . . . . . . . . . . . . 865

26.3 On Rationality in Medicine . . . . . . . . . . . . . 866

26.3.1 Theoretical and Practical Rationality . . . . . . . . 866

26.3.2 Rationality in Medical Sciences . . . . . . . . . . . . 867

26.3.3 Rationality in Clinical Practice. . . . . . . . . . . . . . 869

26.3 .4 The Relativity of Rationality $\ldots \ldots \ldots \ldots \ldots \ldots \ldots . \ldots . \ldots$

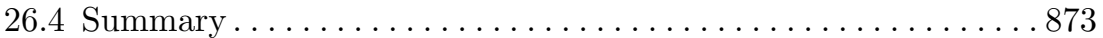

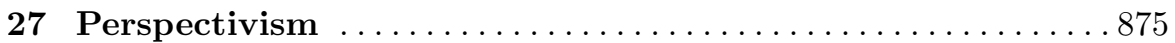

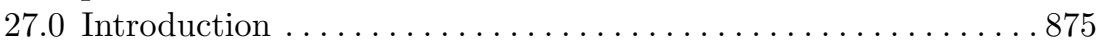

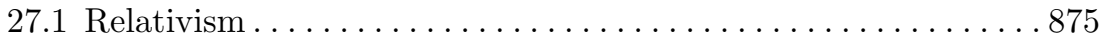

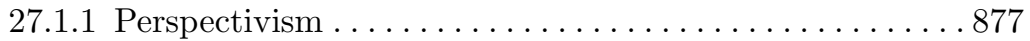

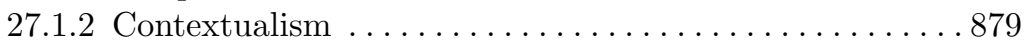

27.2 Perspectivism de re and Perspectivism de dicto ..........880

27.3 Summary.............................. 884 


\section{Part IX Logical Fundamentals}

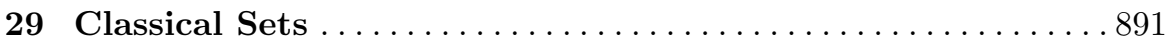

29.0 Introduction . . . . . . . . . . . . . . . . . . . . . . . . . . . . 891

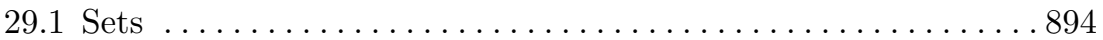

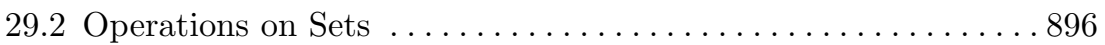

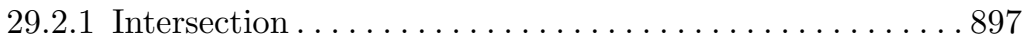

29.2 .2 Union . . . . . . . . . . . . . . . . . . . . . . . . . 897

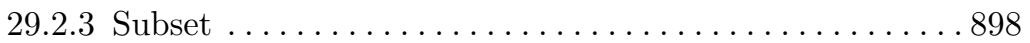

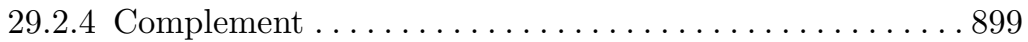

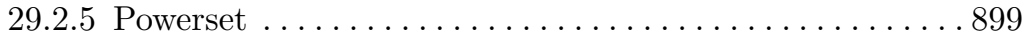

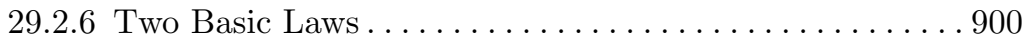

29.3 Relations . . . . . . . . . . . . . . . . . . . . . 901

29.3.1 Ordered Tuples . . . . . . . . . . . . . . . . . . 901

29.3.2 Cartesian Products . . . . . . . . . . . . . . . . . 903

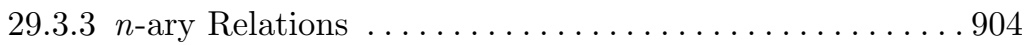

29.4 Functions. . . . . . . . . . . . . . . . . . . . . 906

29.4.1 Functions are Single-Valued Relations . . . . . . . . . . 906

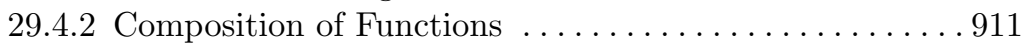

29.4.3 Restriction of a Function . . . . . . . . . . . . . . 913

29.4.4 Point and Set Functions . . . . . . . . . . . . . . . . 913

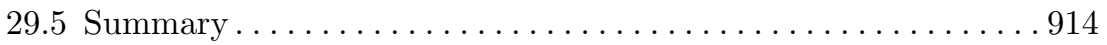

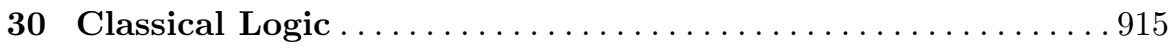

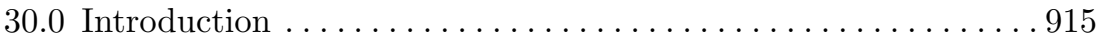

30.1 Basic Concepts . . . . . . . . . . . . . . . . . . . . . . . . 917

30.1.1 Reasoning, Argumentation, and Proof . . . . . . . . . . 917

30.1 .2 The Classical Concept of Inference . . . . . . . . . . . . . 919

30.1.3 Object Language and Metalanguage . . . . . . . . . . . . . 922

30.1.4 Syntax, Semantics, and Pragmatics ............... 923

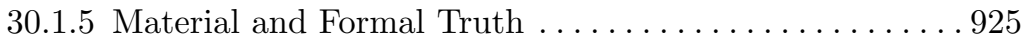

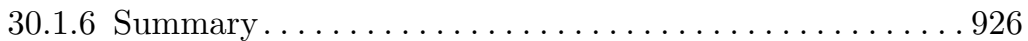

30.2 Classical First-Order Predicate Logic with Identity . . . . . . . . 927

30.2 .1 The Syntax of the Language $\mathcal{L}_{1} \ldots \ldots \ldots \ldots \ldots 27$

30.2 .2 The Semantics of the Language $\mathcal{L}_{1} \ldots \ldots \ldots \ldots \ldots 43$

30.2 .3 A Predicate-Logical Calculus . . . . . . . . . . . . . 960

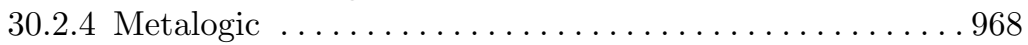

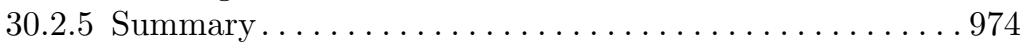


31 Modal Extensions of Classical Logic . . . . . . . . . . . . . . 977

31.0 Introduction . . . . . . . . . . . . . . . . . . . . . . . . . . . . . . 977

31.1 Alethic Modal Logic . . . . . . . . . . . . . . . . . . . . . . 983

31.1.1 Alethic Modalities and Operators............... 983

31.1.2 A First-Order Alethic Modal Logic . . . . . . . . . . . . . . . 985

31.1.3 Metalogic . . . . . . . . . . . . . . . . . . . . . . . 992

31.1.4 Necessary vs. Contingent Identity . . . . . . . . . . . . 992

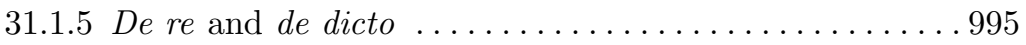

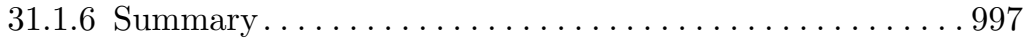

31.2 Deontic Logic . . . . . . . . . . . . . . . . . . . . . . 997

31.2.1 Deontic Modalities and Operators .............. . 998

31.2.2 The Standard System of Deontic Logic . . . . . . . . . . . . . 999

31.2 .3 Metalogic . . . . . . . . . . . . . . . . . . . . . 1003

31.2.4 Deontic Conditionals. . . . . . . . . . . . . . . . . . . . 1005

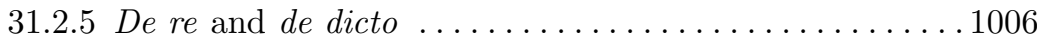

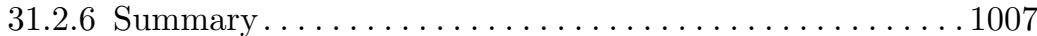

31.3 Epistemic Logic . . . . . . . . . . . . . . . . . . . . . . . . . . . 1007

31.3.1 Epistemic Modalities and Operators . . . . . . . . . . . 1008

31.3.2 A First-Order Epistemic Logic . . . . . . . . . . . . . . . . . 1010

31.3 .3 Metalogic . . . . . . . . . . . . . . . . . . . . 1013

31.3.4 Opaque Epistemic Contexts . . . . . . . . . . . . . . 1014

31.3.5 De re and de dicto ........................ . . . . . . . . . . . . . . . . . . . . . .

31.3.6 Dynamic Epistemic Logic . . . . . . . . . . . . . . . . . . . . 1017

31.3 .7 Summary . . . . . . . . . . . . . . . . . . . . . . . . . . . . . . . . . . . . . . . . 1019

31.4 Temporal Logic . . . . . . . . . . . . . . . . . . . . . . . . . . 1019

31.4.1 Temporal Modalities and Operators . . . . . . . . . . . 1020

31.4.2 A Minimal System of Temporal Logic . . . . . . . . . . . . . . 1021

31.4 .3 Metalogic . . . . . . . . . . . . . . . . . . . . . . . . 1024

31.4 .4 Since and Until . . . . . . . . . . . . . . . . . . . . . . . 1025

31.4.5 Metric Temporal Logic . . . . . . . . . . . . . . . . . . . . . . . . . . . . . . . . 1027

31.4.6 Alternative Approaches . . . . . . . . . . . . . . . . . . . . . . . . . . . . . . . . . . . . . . .

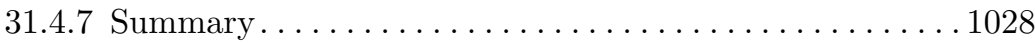

32 Non-Classical Logics . . . . . . . . . . . . . . . . . . . . . . . . . . 1029

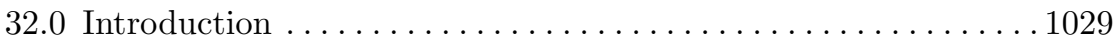

32.1 Relevance Logic . . . . . . . . . . . . . . . . . . . . . . . . . . 1030

32.2 Intuitionistic Logic . . . . . . . . . . . . . . . . . . . . . . . . . . . 1031

32.3 Paraconsistent Logic . . . . . . . . . . . . . . . . . . . . . . 1031

32.4 Non-Monotonic Logic . . . . . . . . . . . . . . . . . . . . . . . . . . 1033

32.5 Many-Valued Logic . . . . . . . . . . . . . . . . . . . . . . . . . . 1034

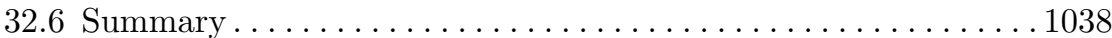




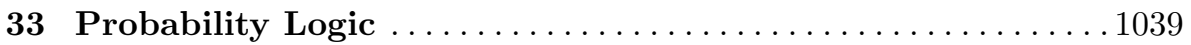

33.0 Introduction . . . . . . . . . . . . . . . . . . . . . . . . . . . . . 1039

33.1 Probability Theory . . . . . . . . . . . . . . . . . . . . . . . . 1040

33.1 .1 Probability Space . . . . . . . . . . . . . . . . . . . 1043

33.1.2 Probability Distribution . . . . . . . . . . . . . . . . . . 1046

33.1.3 Probabilistic Independence . . . . . . . . . . . . . . . . . . . 1048

33.1.4 Conditional Probability . . . . . . . . . . . . . . . . . . . . 1048

33.1.5 Bayes's Theorem . . . . . . . . . . . . . . . . . . . 1050

33.1.6 What Does "Probability" Mean? .................. 1053

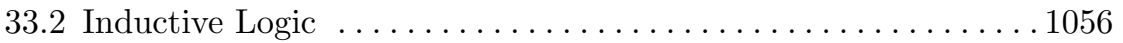

33.3 Bayesian Logic . . . . . . . . . . . . . . . . . . . . . . . . . . . . . . . . . . . . . . . . . . . . . . . . . . . . . . .

33.4 Summary . . . . . . . . . . . . . . . . . . . . . 1063

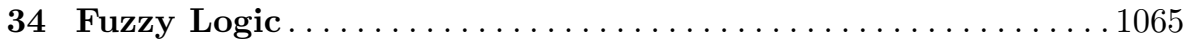

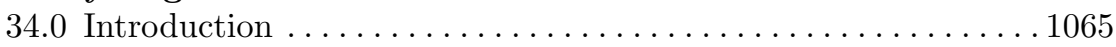

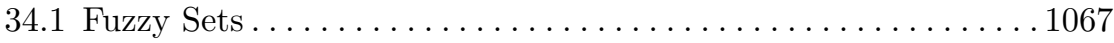

34.2 Operations on Fuzzy Sets . . . . . . . . . . . . . . . . . . . . . 1076

34.2.1 Fuzzy Complement . . . . . . . . . . . . . . . . . . 1077

34.2.2 Fuzzy Intersection and Union . . . . . . . . . . . . 1078

34.2.3 Empty Fuzzy Set and Fuzzy Powerset . . . . . . . . . . . . . 1079

34.2.4 Degrees of Fuzziness and Clarity . . . . . . . . . . 1079

34.2.5 Fuzzy Logic is a Non-Classical System . . . . . . . . . . . . . 1084

34.3 Fuzzy Relations . . . . . . . . . . . . . . . . . . . . . . . . . 1084

34.3.1 The Concept of a Fuzzy Relation . . . . . . . . . . . . . . 1085

34.3.2 Composition of Fuzzy Relations . . . . . . . . . . . . . 1087

34.4 Fuzzy Logic Proper . . . . . . . . . . . . . . . . . . . . . . . . . . . 1090

34.4.1 Linguistic and Numerical Variables ................ 1091

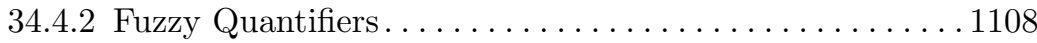

34.4.3 Fuzzy Sentences . . . . . . . . . . . . . . . . . . . . . . . . . . . . . . . . . 1109

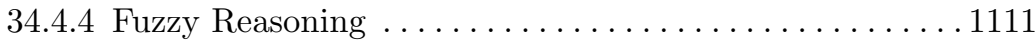

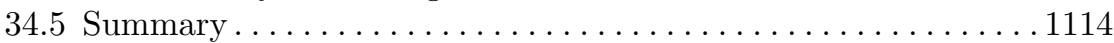

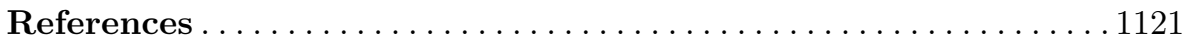

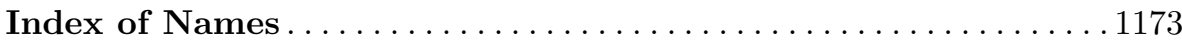

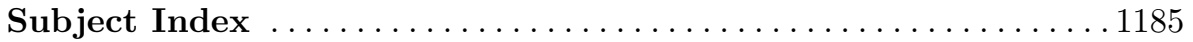




\section{Introduction}

In a pain there is always more knowledge about the truth than in all wise men's serenity. All I know I have learned from the unfortunates, and what I recognized I saw through the look of the pained (Stefan Zweig, 1922, [1993] 56, translated by the present author).

\subsection{A Fresh Start}

I invite readers to look with me at medical practice and research to identify their philosophical, methodological, and logical problems with the purpose of trying to understand what is going on in health and patient care and to find out how to enhance their quality. Let me explain:

Errors of diagnosis and treatment are major problems in health care, despite recent advances in biomedical and clinical sciences and technology. They are due to physician fallibility, on the one hand; and medical imperfection, on the other, raising the question of how the failures emerge and whether it is possible to prevent them. To evaluate this question and to understand its far-reaching implications, we may first consider the following five examples: ${ }^{1}$

1. A 42-year-old female teacher consulted her family physician because of diarrhea that had lasted for five days. The doctor diagnosed enteritis and administered antibiotics. The patient died the next week. An autopsy revealed that she had a stomach cancer.

2. A 49-year-old male physiologist had been suffering from some malaise for several weeks. Based on his own expert knowledge, he convinced himself that he had exocrine pancreatic insufficiency, i.e., lowered production of digestive enzymes by pancreas. He visited an internist to have his suspicion examined. The doctor took some blood tests. A few days later, she calmed the patient down assuring him he did not have an exocrine pancreatic insufficiency. Since his health didn't improve, in the years that followed he successively consulted five additional doctors, to receive additional, conflicting diagnoses. It was only the last, sixth, doctor who was able to confirm his own, initial suspicion of suffering from exocrine pancreatic insufficiency, and to help him.

3. A 56-year-old housewife complained of being poisoned by her neighbors, and was hospitalized in a psychiatric institution. Paranoid schizophrenia was

${ }^{1}$ The examples 2 and 3 are real patient histories encountered by the author himself.

The other three are based on (Cutler, 1998). 
diagnosed. In the third year of her hospital life, a new, young doctor at the ward discovered that the patient had cancer of the esophagus. He concluded the cancer had certainly existed, at least as a precancer, prior to the patient's hospitalization three years earlier, and had caused her gastro-esophageal distress that she had interpreted as a symptom of being poisoned by her neighbors. But it was now too late to correct the past. She died shortly after the diagnosis was made.

4. A 22-year-old female student was diagnosed of having multiple sclerosis because of her complaints of permanent, unbearable headaches, and of some sensory and muscular problems. She was treated for multiple sclerosis over the next eight years. After she moved to another city and changed her apartment, her health problems disappeared immediately. No further treatment was needed. She was able to continue her university studies. Her new doctor speculated that effluents from the furniture in her previous apartment might have been the cause of her health problems.

5. A 39-year-old male engineer had several episodes of sharp, stabbing left chest pain. The pain lasted only a few minutes each time, did not radiate, and was not related to physical activity. The physician whom he visited, found that his ECG was normal and all blood parameters, including blood lipids, were also normal. No risk factors were present. The patient had no history of any disease. X-rays of thoracic organs displayed no abnormalities. The doctor diagnosed Tietze's syndrome and sent the patient home, assuring him that he had no serious health problem. He was asked to return in six weeks. Two weeks later the physician read his obituary notice in the local newspaper. He had not survived a second heart attack.

There are still many more misdiagnoses, wrong treatments, and physiciancaused misfortunes, pains, and deaths. Why and how do they arise? I have tried to understand this phenomenon since the beginning of my clinical training in 1968. Living in West Berlin then, i.e., the free sector of then divided Berlin, Germany, I regularly witnessed at clinical rounds the debates between our chief and senior officers about their conflicting bedside diagnoses and treatment recommendations. It was surprising and even disturbing to me as a young physician to encounter such differences among their clinical judgments. This observation made me aware of an issue for the first time that our teachers had not taught us during our medical education, i.e., methods of clinical reasoning. Clinical reasoning, also called clinical decision-making, diagnostic-therapeutic decision-making, clinical judgment, and clinical cognition, lies at the heart of clinical practice and thus medicine. Although as students of medicine we had learned large parts of natural sciences such as chemistry, physics, and biology; of biomedical sciences auch as anatomy, physiology, biochemistry, pathology, and pathophysiology; and many clinical disciplines, diseases, therapies, and disease-specific methods of diagnosing and treating individual diseases such as gastritis, leukemia, schizophrenia, etc., we had learned nothing about how to search for a diagnosis and treatment in general, i.e., how to arrive at a clinical judgment. I asked myself whether there was a scientific methodology of 
clinical judgment that our teachers had withheld from us, and if so, what did it look like? My extensive search was disappointing. It revealed that there was no such methodology. I have since been concerned with this topic, and have found that a variety of highly intriguing linguistic, logical, methodological, mathematical, epistemological, moral, and metaphysical issues and problems are involved. The present book addresses these issues and problems, many of which have either been overlooked or neglected until now by both medicine and its philosophers. Their analysis will not only enrich medical practice, research, and philosophy, but may also stimulate interest in the other areas involved.

\subsection{The Objective}

Medicine constitutes one of the major and most influential social institutions, including religion, law, education, and government, that interpret, rule, and shape our lives. It is therefore desirable to examine the adequacy and quality of its methods, means, practices, and perspectives. The present book undertakes such an examination by inquiring into the structure, nature, and goals of medicine. Our aim is to clarify the conceptual, methodological, epistemological, moral, logical, and metaphysical foundations of medicine in order to understand what occurs in the doctor-patient clinical encounter; what factors, forces, and sciences determine the dynamics and products of this interaction system; and how to best organize it.

\subsection{The Subject}

To attain our above-mentioned goals, we shall do analytic philosophy of medicine. But what is analytic philosophy of medicine?

Analytic philosophy that has emerged at the turn of the 20th century, is a well-established method of philosophical inquiry by means of logical and conceptual analysis. It was founded by the German mathematician and logician Gottlob Frege (1848-1925), and the British mathematician and logician Bertrand Russell (1872-1970). ${ }^{2}$ It attempts to clarify the structure and meaning of concepts, conceptual systems, knowledge, and action, and to analyze and improve methods of scientific investigation and reasoning. Accordingly,

\footnotetext{
${ }^{2}$ It is sometimes maintained in the literature that analytic philosophy was founded by the British philosopher George Edward Moore (1873-1958) and the AustrianBritish philosopher Ludwig Wittgenstein (1889-1951). However, it began earlier in Friedrich Ludwig Gottlob Frege's works on the philosophy of mathematics and language (Frege, 1884, 1891, 1892a, 1892b, 1893, 1904; Kenny, 2000; Beaney, 2013a), which caused Bertrand Arthur William Russell to change his neo-Hegelian perspective (Russell, 1969) and initiate the logical phase of his philosophical inquiries as of 1900 (Russell, 1903, 1905, 1914, 1919; Whitehead and Russell, 1910).
} 
analytic philosophy of medicine is philosophy of medicine by means of logical and conceptual analysis (Sadegh-Zadeh, 1970a-c, 1977c).

My basic motive for analyzing medicine logically is my long-standing interest in the sources and conundrums of physician fallibility and medical imperfection; my desire to contribute to enhanced physician performance; and my conviction that such enhancement is feasible by employing logic in medicine. A measure of physician performance is provided by the quality of diagnostictherapeutic decisions. Since these decisions are obvious outcomes of clinical reasoning, their quality mirrors the quality of that reasoning. It is well known, however, that despite the advances in medical science and technology, many clinical decisions turn out wrong, leading to malpractice suits. As some statistic report, there are 30-38\% misdiagnoses (Gross and Löffler, 1997; SadeghZadeh, 1981c). At first glance, these errors call into question the clinical competence of the physicians involved. Viewed from a practical perspective, this deficiency in physician performance may appear as a failure that in principle is avoidable by improving the diagnostic-therapeutic methodology, say for example, using 'medical expert systems'. However, there are also scholars who interpret it as an inevitable physician fallibility due to the peculiarity of clinical practice as "a science of particulars" (Gorovitz and MacIntyre, 1976).

I have been systematically concerned with the issues surrounding clinical reasoning and its imperfection since about 1970 . In the present book, some results of this endeavor are discussed. They reveal the deeply philosophicalmetaphysical character of medicine, the realization of which is likely to exert far-reaching impacts on both medicine and philosophy of medicine. The discovery that was briefly mentioned on page $\mathrm{X}$ in the Preface to the First Edition, represents one of them. That is, (i) medicine as health care consists of obligatory well-doings and avoiding prohibited wrong-doings, and is thus practiced morality; and (ii) as clinical research, it seeks, justifies, and establishes rules of that practice, and thus, belongs to normative ethics (SadeghZadeh, 1983). In contrast to the philosophically and methodologically sterile debate about whether medicine is a science or an art (Montgomery, 2006; Munson, 1981), the above thesis asserts that medicine is a duty-driven and normative, i.e. deontic, discipline. (The adjective "deontic" originates from the Greek term $\delta \varepsilon \varepsilon^{\circ}$, deon, for "what is binding", "duty".) I am confident that philosophers of medicine as well as medical professionals will welcome this surprising finding. As we shall see later, its recognition and understanding requires minutely detailed logical analyses of medical language, concepts, knowledge, and decisions. The logic primer provided in the final part of the book is meant to make such illuminating analyses possible. Apart from its philosophical-metaphysical fertility, the finding will also stimulate medical informaticians and expert system researchers to customize their clinical decisionsupport systems and hospital information systems accordingly, and to base them on deontic logic. (For deontic logic, see Section 31.2 on page 997.)

The book is divided into nine parts, Parts I-IX, comprising 34 chapters. The starting-point is the patient, examined in Part II, since the philosophy of 
medicine that I shall develop will be tailored to her/his needs and interests. To this end, in the opening Part I preceding it (pp. 13-118), the language of medicine is carefully studied. It is shown at the outset why the choice of a particular language has effects on the knowledge that is expressed by using its vocabulary. This motivates us to thoroughly consider the syntax and semantics of medical language and the problems caused by the ambiguity and vagueness of its terms. The pragmatic aspects of medical language are analyzed to show its deep embeddedness in the social sphere. Also a brief introduction is given to what is called medical linguistics in medical informatics and information sciences, including medical vocabularies, terminologies, and coding systems. Most important for medicine and its philosophy and methodology is the subsequent analysis of the varieties of medical concepts (qualitative, comparative, quantitative, dispositional, classical, non-classical, etc.) and the comprehensive introduction to methods of scientific concept formation in the closing chapter of this Part I.

Part II focuses on medical praxiology, i.e., the theory of medical practice (pp. 121-398). To this end, the patient is interpreted as a bio-psycho-social and moral agent in order to propose a theory of organism, an emergentist theory of psyche, and a concept of sociosomatics that substitutes for psychosomatics. This interpretation will help to provide an understanding of what it means to say that such an agent may feel ill, or be categorized as diseased. In the pursuit of this understanding, the concepts of health, illness, disease, diagnosis, prognosis, therapy, and prevention are logically analyzed, and a number of novel conceptual frameworks are advanced. These include the prototype resemblance theory of disease, according to which a few prototype diseases determine, by similarity relationships, the whole category of diseases; a probabilistic theory of etiology, which reconstructs medical causality as probabilistic-causal associations between cause and effect; and a theory of relativity of clinical judgment, according to which diagnostic-therapeutic decisions and preventive measures are relative to a number of parameters. The aim is to inquire into how medicine is engaged in shaping the human world, by deciding who is a patient to be subjected to diagnostics and therapy, and who is a non-patient. In this way, nosology, pathology, etiology, diagnostics, prognostics, therapy, and prevention are understood as conceptual and methodological endeavors that serve as means of medical worldmaking. All necessary logical tools are provided in our logic primer in Part IX.

Part III is devoted to medical knowledge (pp. 401-572). In it, we analyze the concept and types of medical knowledge to expose the relationships of this knowledge to what it talks about. It is shown that medical knowledge consists of norms, hypotheses, and theories. While mainly for syntactic reasons medical norms and most types of medical hypotheses are not verifiable, theories are empirically not testable at all because, like norms, they do not consist of statements of facts. They are conceptual structures, just like buildings are architectural structures. Several sample theories are reconstructed according to this non-statement view of theories, to discuss its medical-epistemological 
consequences. An important question in this context is from where medical theories and knowledge arise. It is shown that, in contrast to our received views, the primary sources of medical knowledge and theories are medicalscientific communities and not individual scientists. Pronouncements such as "The German physician and becteriologist Robert Koch discovered the bacillus of tuberculosis" are inappropriate because underlying such a discovery are groups of interacting scientists and technical assistants, research funding agencies, and a number of social and political-historical factors. This socialconstructivist idea was first developed by Ludwik Fleck and adopted later by Thomas Kuhn. It is of particular significance in medicine because it implies that, by and large, medical-scientific communities determine the nature of medical truth and the way how to act. We even go one step further to suggest a theory of technoconstructivism, according to which empirical-scientific research today is in transition to engineering; and empirical-scientific knowledge is increasingly being constructed as a technical product and commodity by technology.

In Part IV (pp. 575-603), the concept of medical deontics is introduced to include under this umbrella term all medical research, on the one hand, whose outcome is formulated by deontic sentences, namely ought-to-do rules; and all health-care practices that obey such deontic rules, on the other. Thus, medical deontics not only covers normative medical ethics and law, but also diagnostic-therapeutic research as well as clinical practice. This momentous deonticity of medicine also includes the concept of disease, that is argued to be a deontic concept created by common morality in the human society. As was already pointed out above, the deontic character of medicine has been ignored until now. I hope that philosophers of medicine, and medical ethicists as well, will concern themselves with this intriguing feature of health care in order to open new fields of research and to enlarge our understanding of how maladies and healing practices and professions are intertwined with morality and charity.

Part V (pp. 607-706) deals with the roles that systems of logic play in medicine, and with the question whether there is an inherent logic of medicine. It is shown that, due to the syntactic richness of medical language, different types of logic are required to cope with it in medical research and practice, because it transcends the scope and capabilities of individual logic systems. In this plurality of logics in medicine, an exception is provided by fuzzy logic. Fuzzy logic, also briefly introduced in our logic primer in the final Part IX (pp. 891-1120), is a logic of vagueness, and therefore highly suitable for use in medicine. It is a general enough logic to satisfy almost all logical needs of medicine, and moreover, to serve as an outstanding methodological tool for constructing innovative techniques of problem-solving in research and practice. This has been demonstrated by an extensive application of fuzzy logic to clinical, biomedical, conceptual, medical-deontic, and metaphysical issues. By virtue of its wide applicability, strength, and elegance, it is likely to become the leading logic in medicine in the not-too-distant future. Besides the 
logical pluralism referred to above, no other logical peculiarity of medicine is observed that would require a specific medical logic. However, that does not mean that there is no rationale behind medical thinking and acting.

In Part VI (pp. 709-743), a brief sketch is given of what has come to be known as artificial intelligence in medicine, 'AI in medicine' or 'AIM' for short. The aim is to analyze the foundations of this new field of research and practice in medicine to understand the major role the innovative technology of artificial intelligence currently plays, and will in the future play, in health care. To this end, the origin of AI in medicine is traced to provide some insights into the development of 'medical decision-making' as a scholarly field of inquiry in close relationship with the emergence of electronic computers around 1950. It is shown why and how AI in medicine branched from medical decision-making. The evolution and prospects of the discipline are outlined to prepare the critical discussion of its impact on the healing relationship and health care in Section 25.7, Quo Vadis Medicina?, on pages 845-852.

Medical metaphysics is the subject of Part VII (pp. 747-852). It is primarily concerned with medical ontology, medical truth, and the nature of medicine:

(i) The basic science ontology is divided into pure ontology, applied ontology, and formal ontology which are thoroughly discussed. In these three areas, novel suggestions have been put forward by using fuzzy logic. Specifically, we have introduced a fuzzy ontology that seems to be auspicious for both medicine and philosophy. It not only determines degrees of being by means of a fuzzy existence operator, that we have dubbed the Heraclitean operator, but also makes it possible to construct a fuzzy mereology, by means of which vague part-whole relationships become tractable. Of particular importance is our distinction between de re and de dicto ontology, that is based on a syntactic criterion, and enables differentiation between fictional entities such as Sherlock Holmes, and real ones. The salient advantage of this approach is that it allows precise analyses of controversial questions like "are diseases fictitious or real?". Using this approach, we have extensively examined the ontological problems associated with nosology, psychiatry, and psychosomatics, and have also critically explored the so-called biomedical ontology engineering that is expanding in medical information sciences today.

(ii) Regarding medical truth, it has been shown that there is sufficient evidence to support the assertion that medical truths are system-relative, and are formed within the respective health care systems themselves. They do not mirror or report scientifically discovered facts 'in the world out there' which could be independent of the conceptual system from where they are viewed and judged. This problem not only concerns truth in medical sciences (p. 819 ff.), but also truth in clinical practice. Specifically, the concept of misdiagnosis has been precisely defined that enables us to discern the system relativitity of misdiagnoses and to identify their causes (p. $821 \mathrm{ff}$.).

(iii) Particular emphasis has been placed on the analysis of medicine as a scientific field. Abandoning widespread, exclusive mono-categorizations such 
as "medicine is a science" versus "medicine is an art", we have demonstrated that in declarations of the type "medicine is such and such", the global term "medicine" should be differentiated to recognize that medicine, comprising many heterogeneous disciplines, belongs to a large number of categories. For example, without doubt biomedicine is natural science; clinical research, however, is practical science; it is also normative ethics; clinical practice is practiced morality; and so on. What is worth noting, is that medicine is also a poietic science (from the Greek term $\pi \mathrm{o} \iota \eta \sigma \iota \varsigma$, "poiesis", for making, creating) that invents, designs, and produces medical devices in the widest sense of the term "making", from drugs to prosthetics to brain chips to artificial organs to artificial babies. Medicine is thus on its way to become an engineering science, conducted as health engineering and anthropotechnology.

Part VIII of the book, an Epilog (pp. 855-888), attempts to clarify some epistemological and metaphysical issues that our preceding analyses of medicine have revealed. First, taking into account the peculiarities of medicine, the concept of science is explicated to demonstrate why the traditional understanding of this concept in the general philosophy of science is terribly one-sided. The yield is a tripartite concept of science that, in contrast to the traditional mono-scientism, suggests three different types of science: theoretical science, practical science, and deontic science. Medicine comprises all three types of science. Second, it is shown that rationality cannot be a criterion of the scientificity of medicine, because rationality is something relative, and depends on the perspective from which it is judged. Third, it is argued that this dependence on perspective is an inescapable property of judgments, rendering perspectivism an interesting approach to the philosophy of worldviews, including epistemology and ontology both in medicine and elsewhere.

To conduct the studies sketched above, we must first assemble the logical and conceptual tools that we shall use in the book. This task is accomplished in Part IX as a sort of Appendix (pp. 891-1120). It provides the logical fundamentals comprising an outline of the relevant systems of logic from classical set theory and logic, to modal logics, non-classical logics and probability logic, and further on to fuzzy set theory and logic. For readers not acquainted with logic and its terminology, Part IX is the prerequisite for understanding the medical-philosophical frameworks and theories developed in the book.

\subsection{Methods of Inquiry}

It is a truism that a tool for analyzing a particular object should be sufficiently sensitive to the subtleties of that object. Otherwise, the details and peculiarities of the analysandum will be lost. For example, it would be fatuous if someone tried to examine a biological cell by employing a pneumatic hammer, since such a brute-force approach only destroys the cell. The ingenious apparatus of a microscope and thin light waves will be necessary to discern what is before one's eyes. The same holds for analyzing a scientific enterprise 
itself. Medicine as a scientific enterprise is too complex an area to be amenable to coarse, crude, confused, or anarchic tools and techniques of inquiry.

My interest in the subject addressed in this book goes back to my youth when, in the early 1960 s, I was a graduate student of medicine and philosophy at the University of Münster in Germany. Initially, I was an adherent to phenomenologic and hermeneutic approaches, until analytic philosophy persuaded me in the late 1960s that it was a more adequate and superior method of philosophical analysis in medicine. I had the good fortune to realize early on that in philosophizing on topics such as diagnostic-therapeutic reasoning or the conceptual structures of medical knowledge, hypotheses and theories, well-developed, sensitive, and precise tools and techniques such as logic are required. The reason why logic is needed rather than a pneumatic hammer for such an inquiry, is simply that both diagnostic-therapeutic reasoning and epistemic entities and structures have some logical characteristics, which are not discernible and adequately analyzable by tools other than logic. Accordingly, my approach to philosophy of medicine in this book takes an analytic route. It is my conviction that medicine will only benefit from logical self-analysis.

\subsection{How to Read this Book}

The subject of the book outlined in Section 0.3 above requires the application of a number of different logics. As a consequence of using logic as our method of inquiry, the book is not light reading like a traditional medical-philosophical treatise. Some knowledge of logic is necessary for understanding the analyses, reconstructions, and constructions involved. Since it would be unrealistic to

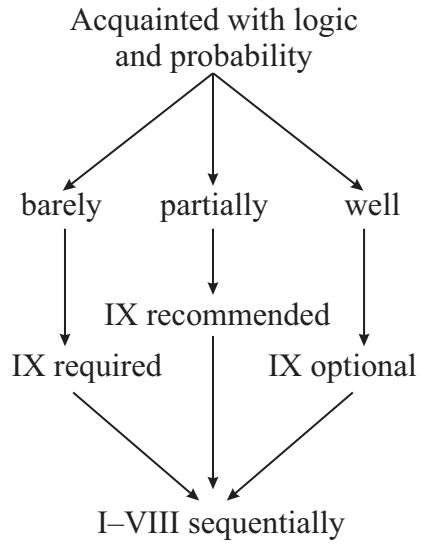

Fig. 1. How to read this book suppose that every reader is acquainted with all the 15 logics used in the book, or to require that she study 15 separate logic primers of about 200 pages each before studying the present book, a concise and easily digestible introduction to their essentials is provided in the final part of the book that she may consult before or while reading chapters of the book. In that case, the book will be absolutely self-contained and easily accessible to any interested student. The author recommends that you study the logic primer in Part IX first, and then proceed sequentially through Parts I-VIII and not skip anything. The book has been organized systematically and should be read accordingly (see Figure 1). 
An important note: It is worth emphasizing at the outset that whenever and wherever we speak of "scientific knowledge" in this book, we mean empirical-scientific, including medical, knowledge. Unless specified otherwise, we never mean formal-scientific, i.e., mathematical or logical, knowledge which may be analytically true or false, but has nothing to do with experiential states of affairs 'in the world out there'. 